\title{
Fatores associados ao comportamento da população durante o isolamento social na pandemia de COVID-19
}

\author{
Factors associated with people's behavior in social isolation during \\ the COVID-19 pandemic
}

Anselmo César Vasconcelos Bezerra (https://orcid.org/0000-0003-0722-9417) ${ }^{1}$ Carlos Eduardo Menezes da Silva (https://orcid.org/0000-0003-1156-156X) ${ }^{1}$ Fernando Ramalho Gameleira Soares (https://orcid.org/0000-0001-8244-1800) ${ }^{2}$ José Alexandre Menezes da Silva (https://orcid.org/0000-0003-4603-978X) ${ }^{3}$
${ }^{1}$ Instituto Federal de Educação Ciência e Tecnologia de Pernambuco. Av. Prof. Luís Freire 500, Cidade Universitária. 50740545 Recife PE Brasil. anselmo@recife.ifpe.edu.br ${ }^{2}$ Instituto Brasileiro de Geografia e Estatística. Rio de Janeiro RJ Brasil.

${ }^{3}$ Netherlands Hanseniasis

Relief. Fortaleza CE Brasil.
Abstract This paper presents the results of an opinion poll conducted in Brazil on the perception of social isolation during the COVID-19 pandemic. The questionnaire was prepared on Google Forms, disseminated through social networks, with questions about the socioeconomic profile and factors associated with isolation. A non-probabilistic sample was obtained with 16,440 respondents. Data were analyzed using the Stata 13 software. Social interaction was the most affected aspect among people with higher education and income (45.8\%), and financial problems caused a more significant impact (35\%) among people with low income and education. Those who practice some physical activity showed lower levels of stress $13 \%$, as well as greater normality in sleep $50.3 \%$. People who reported living in worse habitability conditions reported willingness to remain isolated for less time, 73.9\%. Among non-isolated people ( $10.7 \%$ of the total sample), $75.8 \%$ believe that social isolation will reduce the number of victims of COVID-19. We conclude, based on this sample, that the perception about social isolation as a pandemic mitigation action varies by income, education, age, and gender. However, most believe that it is the most appropriate control measure and are willing to wait as long as necessary to contribute to the fight against COVID-19.

Key words New Coronavirus, Brazil, Social perception
Resumo $O$ artigo apresenta resultados da pesquisa de opinião realizada no Brasil sobre a percepção do isolamento social durante a pandemia de COVID-19. O questionário foi elaborado no Google Forms, disseminado por redes sociais, com questões sobre o perfil socioeconômico e fatores associados ao isolamento. Obteve-se uma amostra com 16.440 respondentes. Os dados foram analisados no software Stata 13. O convivio social foi $o$ aspecto mais afetado entre pessoas com maior escolaridade e renda $45,8 \%$, para pessoas de baixas renda e escolaridade, problemas financeiros provocam maior impacto $35 \%$. Os que praticam atividade fisica revelaram menores niveis de estresse $13 \%$, bem como uma maior normalidade no sono 50,3\%. Pessoas que referiram residir em piores condições de habitabilidade, informaram disposição a permanecer menos tempo isoladas $73,9 \%$. Dentre as pessoas que não estão isoladas (10,7\% do total), $75,8 \%$ acredita que o isolamento social reduzirá o número de vítimas da COVID-19. Concluímos, que a percepção das pessoas quanto ao isolamento social como medida de mitigação da pandemia, varia conforme a renda, escolaridade, idade e sexo, porém a maior parte acredita que se trata da medida de controle mais indicada e estão dispostas a esperar o tempo que for necessário para contribuir com o enfrentamento à COVID-19.

Palavras-chave Novo Coronavírus, Brasil, Percepção social 


\section{Introdução}

Em dezembro de 2019 a China informou à OMS sobre um surto de uma nova doença, semelhante a uma pneumonia. Essa doença, transmitida pelo novo coronavírus, foi denominada COVID-19'. Em janeiro de 2020, novos casos da COVID-19 foram notificados fora da China ${ }^{2}$, então a OMS resolveu declarar emergência internacional em saúde pública ${ }^{3}$. Na América Latina, o primeiro caso registrado foi em São Paulo, no Brasil, no dia 26 de fevereiro de $2020^{4,5}$.

Após a chegada da COVID-19 no Brasil, diversas medidas de controle e prevenção da doença foram tomadas pelas autoridades sanitárias locais em diferentes esferas administrativas (governo federal, governos estaduais e municipais). Essas medidas se diferenciaram de uma região para outra do país, entretanto a medida mais difundida pelas autoridades foi a prática do distanciamento social ${ }^{6}$, entendida de forma geral pela população e pela mídia, como isolamento social ${ }^{7}$, por isso nesta pesquisa optou-se por esse termo, considerado o de mais fácil compreensão pelas pessoas.

A prática do isolamento social tem causado muitas polêmicas no país, uma vez que algumas autoridades mostram-se céticas quanto à sua eficácia ${ }^{7}$. O fato é que a maior parte dos tomadores de decisão optaram por incentivar essa medida, adotando estratégias de controle da mobilidade da população, como o fechamento de escolas e universidades, do comércio não essencial, e de áreas públicas de lazer etc. ${ }^{8}$. Como resultado, grande parte da população brasileira apoiou e aderiu ao movimento do isolamento social ${ }^{9} \mathrm{com}$ o objetivo de se prevenir da COVID-19 e de colaborar com a atenuação da curva de contágio no país ${ }^{10}$.

Todavia, o processo de isolamento social tem causado alguns impactos na vida das pessoas ${ }^{11-13}$. Por isso, decidiu-se nesta pesquisa buscar compreender os principais efeitos desse isolamento social, observando os fatores que podem contribuir ou atrapalhar nesse processo, mas também correlacionar algumas características socioeconômicas da população com os fatores associados ao isolamento social.

O objetivo principal desta pesquisa foi descrever, a partir da percepção dos respondentes, aspectos relacionados ao comportamento das pessoas e como estas estão sendo afetadas durante o isolamento social imposto pela pandemia da COVID-19. As dimensões avaliadas levaram em consideração: fatores econômicos (impac- to na renda/gastos), de saúde (nível de estresse, prática de atividade física, qualidade do sono), ambientais (quantidade de pessoas na residência, percepção de conforto da residência, presença de áreas abertas na residência), e ao tempo que as pessoas estão dispostas a se manterem em isolamento no contexto da pandemia.

Existe uma discussão na mídia e no senso comum de que a parcela com menor renda está praticando menos o isolamento social em relação àquela com maior renda, principalmente em função da necessidade de locomoção para o trabalho, uma vez que a população mais pobre está vinculada a atividades essenciais que não pararam, e a população com maior renda está, de forma geral, mais vinculada às atividades que pararam e/ou estabeleceram o trabalho remoto. Outros fatores que interferem no isolamento social também são questionados pela mídia e pela academia, como, por exemplo, as diferenças nas condições de habitabilidade entre as pessoas de maior e menor renda. Diante disso, a pesquisa procurou descrever se esses fatores estão coerentes com a percepção da população sobre o processo de isolamento social.

Pesquisas semelhantes já foram desenvolvidas em outros países. Na Itália, por exemplo, identificou-se que a população fica menos disposta a colaborar com o auto isolamento se as medidas de extensão desse isolamento forem prorrogadas ao longo do tempo ${ }^{14}$. No Reino Unido, o desejo pelo autoisolamento foi alto em todos os segmentos sociais, entretanto as pessoas de menor renda apresentaram três vezes menos chances de praticar o autoisolamento ${ }^{15}$, especialmente em função do tipo de trabalho exercido.

No Brasil, algumas pesquisas sobre o isolamento social já foram divulgadas ${ }^{9,16}$. O Datafolha ${ }^{9}$ evidenciou que $76 \%$ dos brasileiros eram a favor do isolamento no início do mês de abril. No Estado do Ceará, evidenciou-se que as medidas de isolamento adotadas pela população possuem variações em função da renda, sexo e escolaridade da população. Na pesquisa, constatou-se que os jovens estão mais vulneráveis à contaminação pela COVID-19, pois estão menos isolados que os idosos ${ }^{16}$.

Apesar dos resultados apresentados neste artigo revelarem somente a percepção dos respondentes da pesquisa e não da população como um todo, diante da urgência imposta pela pandemia para a ampliação da base de conhecimento comum relacionada às estratégias para enfrentá-la, as considerações aqui apresentadas podem lançar luzes sobre questões relevantes ao desenho de es- 
tratégias para reduzir problemas que o isolamento social pode causar em diferentes segmentos da sociedade. Ressaltando que a literatura aponta que o isolamento social é uma das principais medidas não farmacológicas para o enfrentamento da pandemia da COVID-19 19 .

\section{Métodos}

Trata-se de um estudo transversal realizado a partir de um questionário com dezessete perguntas objetivas no formato de pesquisa de opinião, conforme normas da resolução $510 / 16^{17}$. Não foi necessário nenhum tipo de identificação dos respondentes e a participação foi voluntária. A pesquisa reuniu dados em meio ao universo da população brasileira, mais especificamente entre aquela que dispõe de algum equipamento digital com acesso à internet, configurando uma amostra não probabilística com viés de conveniência.

O questionário foi estruturado em quatro partes, para identificar o máximo de dados relacionados aos objetivos ${ }^{18}$.

1) Questões referentes ao perfil socioeconômico dos respondentes, contendo as variáveis de sexo, idade, estado de residência, escolaridade e faixa de renda.

2) Perguntas referente ao isolamento e ao impacto dele na vida das pessoas com as seguintes variáveis: se está ou não isolado, por que não está isolado, qual o principal impacto do isolamento, como o isolamento está afetando a renda/gastos e a saúde.

3) Questões referentes às condições de habitabilidade no isolamento: número de pessoas na residência, percepção de conforto da residência, presença ou ausência de área aberta e/ou verde na residência.

4) A última pergunta foi sobre a expectativa das pessoas em relação ao tempo máximo que acreditam suportar na condição de isolamento social durante a pandemia.

O instrumento de coleta foi construído na plataforma Formulários Google ${ }^{\circledR}$ e divulgado via internet, através dos aplicativos e redes sociais: whatsapp, instagram e facebook, entre os dias 6 e 8 de abril de 2020. Ao todo, obtiveram-se 17.254 respostas de todos os estados brasileiros, com diferentes proporções no número de respondentes. Só foram consideradas as respostas de pessoas com dezoito anos ou mais, o que reduziu o número de observações para 16.440 .

Os dados foram tabulados em planilha de Excel e analisados por meio do software STATA ${ }^{\circledR} 13$.
Foi aplicado para cada relação entre variáveis categóricas o teste Qui-quadrado de Pearson, o qual calcula, além do valor da variável qui-quadrado, o p-valor dessa amostra. $O$ teste verifica se existe associação entre variáveis categóricas, sendo possível refutar ou não a hipótese nula de independência. Neste artigo, adotou-se como parâmetro para refutar a hipótese nula, após a exploração de diferentes variáveis com diferentes graus de liberdade, um nível de confiança desejável de 99\% para análise do valor crítico da distribuição quiquadrado, o que exige para identificar associação um p-valor maior que 0,01 (nível de significância de $1 \%)$.

\section{Resultados}

Do total de 16.440 respostas válidas da amostra, $69 \%$ das pessoas eram do sexo feminino, enquanto $31 \%$ do masculino. Em relação às faixas de renda, observou-se que $34 \%$ das respostas estava na faixa de até 2 salários mínimos, $31 \%$ na faixa que recebe entre 2 e 5 salários mínimos, 17\% recebem entre 5 e 8 salários mínimos e 19\% ganham acima de 8 salários mínimos.

Sobre escolaridade, a pesquisa não pretendia atingir nenhum segmento social em específico, entretanto os dados revelaram uma concentração de respostas de pessoas com ensino superior (34\%) e pós-graduação (52\%). Enquanto $13 \%$ dos respondentes possuem ensino médio e $1 \%$ ensino fundamental. A variação média da idade dos respondentes foi de 41 anos e a mediana de 40 anos. Os percentuais de respostas aumentaram a partir dos 30 anos e diminuíram após os 55 anos.

No universo amostrado, 32\% afirmaram que estão em isolamento total, ou seja, não saem de suas residências; $57 \%$ das pessoas estão em isolamento parcial, termo que significa, nesta pesquisa, sair de casa apenas para comprar alimentos e medicamentos; e $11 \%$ das pessoas não se enquadram nem como isoladas nem como parcialmente isoladas. De forma geral, $89 \%$ das pessoas acreditam que o isolamento reduz o número de vítimas da COVID-19, enquanto $8 \%$ não têm certeza, e apenas 3\% responderam que o isolamento não é capaz de reduzir o número de vítimas da COVID-19.

Para 39\% dos respondentes, o convívio social é o principal aspecto que está sendo afetado pelo isolamento, já 24\% das pessoas relataram o aspecto financeiro como o mais impactante, 19\% dos respondentes informam que o isolamento 
não está ocasionando nenhum tipo de impacto e $10 \%$ apontam outros fatores que estão sendo impactados, apenas $8 \%$ atribuem o principal impacto à própria saúde. Os fatores listados podem estar inter-relacionados, mas a pergunta objetivou compreender qual o impacto principal, mesmo se a pessoa estiver sendo impactada por mais de um fator. Observou-se que para esta variável, quando estratificada por perfil de renda, os resultados mostram diferenças significativas entre os grupos.

Quando questionou-se sobre o impacto do isolamento social na renda/gastos das pessoas, as respostas foram as seguintes: para 32\% das pessoas, o isolamento não está impactando a renda/ gastos, já 34\% dos respondentes afirmam que estão economizando dinheiro, $13 \%$ das pessoas estão gastando mais dinheiro nesse período, enquanto $20 \%$ afirmaram que pararam de ganhar dinheiro em função do isolamento social.

Quando inquiridos se o isolamento estava gerando algum estresse no ambiente doméstico, $27 \%$ disseram que não estavam sentindo nenhum estresse em função do isolamento, 56\% relataram estar sentindo um pouco de estresse e $17 \%$ afirmaram que o isolamento tem gerado muito estresse no ambiente doméstico.

Investigou-se também, como está o sono das pessoas diante desse momento de isolamento social. Dos respondentes, $44 \%$ afirmaram que estão mantendo o número de horas de sono que já dormiam antes do isolamento. Entretanto, 56\% relataram alguma modificação nas horas de sono, decompondo-se em: 26\% que estão dormindo mais horas que o habitual e $31 \%$ de pessoas que estão dormindo menos horas. Em relação à atividade física, $40 \%$ das pessoas está fazendo algum exercício e $60 \%$ não.

Em relação às condições da habitação, questionou-se sobre qual o número de pessoas que compartilham a mesma residência junto à pessoa que preencheu o questionário nesse período. $\mathrm{Ob}$ servou-se que a média de pessoas por residência durante o isolamento social no grupo entrevistado é de 3,2. Verificou-se que o maior percentual de respondentes está vivenciando o isolamento em residências com 2 a 4 pessoas, o que representa em grande parte as características do perfil majoritário das pessoas que responderam à pesquisa.

Em relação às condições de habitabilidade, as respostas mostraram que $82 \%$ das pessoas consideram sua residência boa ou ótima em relação aos fatores: tamanho, abastecimento de água e ventilação, enquanto apenas $18 \%$ a consideram como regular, ruim ou péssima. $63 \%$ também responderam que a residência possui alguma área aberta (terraço, quintal, área verde), contra $37 \%$ que não possuem. Dos que responderam que possuem alguma área aberta em casa, 68\% acham que isso ajuda muito a conviver no período de isolamento.

Em relação à expectativa do tempo em que a pessoa acredita que consegue ficar em condições de isolamento social, os dados mostram que $16 \%$ das pessoas afirmam que não conseguem ficar um mês inteiro nesta condição, 20\% responderam que conseguem ficar entre um e dois meses no isolamento, 3\% acreditam que conseguem ficar mais de dois meses, se necessário, porém, a maioria de $61 \%$ está disposta a ficar o tempo que for necessário nesta condição para enfrentar a pandemia.

Os resultados permitiram observar quais os fatores que mais influenciam na percepção da importância do autoisolamento como principal estratégia para o enfrentamento da pandemia. Pouco mais de $10 \%$ dos respondentes não estavam em isolamento, mas mesmo dentre estes, o percentual dos que acreditam na estratégia de isolamento social é maioria 75,79\%.

Dentre os grupos de pessoas que estão em isolamento total e parcial, a imensa maioria, respectivamente, $88,28 \%$ e $93,32 \%$, acredita que o isolamento social contribui para a redução no número de vítimas da COVID-19. No entanto, $7,88 \%$ ainda têm dúvidas sobre a eficácia da estratégia. Dos que apontaram que o principal impacto do isolamento está sendo interromper a sua renda, 79\% acreditam que o isolamento social tem um impacto na redução do número de vítimas da COVID-19.

Observou-se que nas faixas de menor renda encontra-se um maior percentual de pessoas que afirma ter parado de ganhar dinheiro no contexto da pandemia: $35 \%$ entre os que declararam não ter renda, $34,8 \%$ entre os que ganham até 1 salário mínimo e $24,76 \%$ entre os que recebem de 1 a 2 salários mínimos, contrastando com os percentuais obtidos nas faixas de renda mais alta, que variaram entre $17,5 \%$ e $12,7 \%$ (Tabela 1 ).

Ainda sobre aspecto financeiro o isolamento social apresentou uma significante correlação entre a percepção de impacto na renda e a de estresse familiar. Isso fica mais evidente quando estratificado por faixas de renda, onde a percepção do aspecto financeiro é maior para 33\% daqueles sem renda; $42 \%$ para aqueles com renda de até 1 salário mínimo e $31,7 \%$ para pessoas com renda entre 1 e 2 salários mínimos. Já entre aqueles 
com faixas de renda maiores, o principal impacto percebido foi no convívio social, $39,7 \%$ entre aqueles com renda entre 2 e 5 salários mínimos, 45,5\% no grupo entre 5 e 8 salários mínimos e $52 \%$ para os que recebem mais de 8 salários mínimos (Tabela 2).

No que diz respeito à percepção do principal impacto em consequência do isolamento, pessoas do sexo masculino elegeram proporcionalmente mais os de convívio social $(41,2 \%)$ e do aspecto financeiro $(27,2 \%)$, enquanto pessoas do sexo feminino elegeram em menor proporção o convívio social $(38,6 \%)$ e o aspecto financeiro (23\%). Em relação à percepção do impacto na renda, os resultados foram semelhantes. Pessoas do sexo feminino proporcionalmente percebem melhor que estão economizando ou gastando mais, enquanto que as do masculino percebem mais a perda de renda.

Quando relacionado o principal impacto observado pelo isolamento com a percepção de ocorrência de algum tipo de estresse familiar, observa-se que para todos que responderam que estavam percebendo algum impacto, em média, $80 \%$ relataram algum tipo de estresse familiar.

O grupo que respondeu a saúde como principal impacto é também o grupo que relata um maior estresse. Para eles, a ocorrência de estresse

Tabela 1. Pessoas respondentes de 18 anos e mais, por faixa de renda, segundo impacto na renda ou gasto.

\begin{tabular}{|c|c|c|c|c|c|c|c|c|}
\hline \multirow{2}{*}{\multicolumn{2}{|c|}{$\begin{array}{c}\text { Impacto na renda ou } \\
\text { gasto }\end{array}$}} & \multicolumn{7}{|c|}{ Faixa de Renda } \\
\hline & & \multirow{2}{*}{$\begin{array}{c}\text { Sem renda } \\
375\end{array}$} & \multirow{2}{*}{ Até $\mathbf{1}$ SM } & \multirow{2}{*}{$\begin{array}{c}\text { Entre } 1 \text { e } 2 \\
\text { SM } \\
686\end{array}$} & \multirow{2}{*}{$\begin{array}{c}\text { Entre } 2 \text { e } 5 \\
\text { SM } \\
1708\end{array}$} & \multirow{2}{*}{$\begin{array}{c}\text { Entre } 5 \text { e } 8 \\
\text { SM } \\
979\end{array}$} & \multirow{2}{*}{$\begin{array}{c}\text { Mais de } 8 \\
\text { SM } \\
1231\end{array}$} & \multirow{2}{*}{$\begin{array}{r}\text { Total } \\
5300\end{array}$} \\
\hline Não & $\mathrm{n}$ & & & & & & & \\
\hline & $\%$ & $(7,1)$ & $(6,1)$ & $(12,9)$ & $(32,2)$ & $(18,5)$ & $(23,2)$ & $(100)$ \\
\hline \multirow[t]{2}{*}{ Economizando } & $\mathrm{n}$ & 425 & 413 & 823 & 1711 & 1002 & 1232 & 5606 \\
\hline & $\%$ & $(7,6)$ & $(7,4)$ & $(14,7)$ & $(30,5)$ & $(17,9)$ & $(22)$ & $(100)$ \\
\hline \multirow[t]{2}{*}{ Gastando mais } & $\mathrm{n}$ & 128 & 207 & 421 & 766 & 374 & 323 & 2219 \\
\hline & $\%$ & $(5,8)$ & $(9,3)$ & (19) & $(34,5)$ & $(16,9)$ & $(14,6)$ & (100) \\
\hline \multirow[t]{2}{*}{ Parou a renda } & $\mathrm{n}$ & 500 & 504 & 635 & 887 & 381 & 408 & 3315 \\
\hline & $\%$ & $(15,1)$ & $(15,2)$ & $(19,2)$ & $(26,8)$ & $(11,5)$ & $(12,3)$ & $(100)$ \\
\hline \multirow[t]{2}{*}{ Total } & $\mathrm{n}$ & 1428 & 1445 & 2565 & 5072 & 2736 & 3194 & 16440 \\
\hline & $\%$ & $(8,7)$ & $(8,8)$ & $(15,6)$ & $(30,9)$ & $(16,6)$ & $(19,4)$ & (100) \\
\hline
\end{tabular}

Teste Qui-quadrado: $X^{2}=756,997 ; \mathrm{p}<0.001$.

Fonte: Elaboração própria.

Tabela 2. Pessoas respondentes de 18 anos e mais, por faixa de renda, segundo percepção do impacto do isolamento.

\begin{tabular}{|c|c|c|c|c|c|c|c|c|}
\hline \multirow{2}{*}{\multicolumn{2}{|c|}{$\begin{array}{l}\text { Impacto do } \\
\text { Isolamento }\end{array}$}} & \multicolumn{7}{|c|}{ Faixa de Renda } \\
\hline & & \multirow{2}{*}{$\begin{array}{c}\text { Sem renda } \\
259\end{array}$} & \multirow{2}{*}{$\begin{array}{c}\text { Até } \mathbf{1} \text { SM } \\
247\end{array}$} & \multirow{2}{*}{$\begin{array}{c}\text { Entre 1 e } 2 \\
\text { SM } \\
493\end{array}$} & \multirow{2}{*}{$\begin{array}{c}\text { Entre } 2 \text { e } 5 \\
\text { SM } \\
1034\end{array}$} & \multirow{2}{*}{$\begin{array}{c}\text { Entre } 5 \text { e } 8 \\
\text { SM } \\
512\end{array}$} & \multirow{2}{*}{$\begin{array}{c}\text { Mais de } 8 \\
\text { SM }\end{array}$} & \multirow{2}{*}{$\begin{array}{c}\text { Total } \\
3092\end{array}$} \\
\hline Não & $\mathrm{n}$ & & & & & & & \\
\hline & $\%$ & $(8,4)$ & (8) & $(15,9)$ & $(33,4)$ & $(16,6)$ & $(17,7)$ & $(100)$ \\
\hline \multirow[t]{2}{*}{ Convívio social } & $\mathrm{n}$ & 406 & 364 & 782 & 2013 & 1244 & 1666 & 6475 \\
\hline & $\%$ & $(6,3)$ & $(5,6)$ & $(12,1)$ & $(31,1)$ & $(19,2)$ & $(25,7)$ & $(100)$ \\
\hline \multirow[t]{2}{*}{ Financeiro } & $\mathrm{n}$ & 478 & 616 & 815 & 1132 & 485 & 462 & 3988 \\
\hline & $\%$ & (12) & $(15,4)$ & $(20,4)$ & $(28,4)$ & $(12,2)$ & $(11,6)$ & $(100)$ \\
\hline \multirow[t]{2}{*}{ Saúde } & $\mathrm{n}$ & 129 & 94 & 208 & 408 & 220 & 223 & 1282 \\
\hline & $\%$ & $(10,1)$ & $(7,3)$ & $(16,2)$ & $(31,8)$ & $(17,2)$ & $(17,4)$ & $(100)$ \\
\hline \multirow[t]{2}{*}{ Outro } & $\mathrm{n}$ & 156 & 124 & 267 & 485 & 275 & 296 & 1603 \\
\hline & $\%$ & $(9,7)$ & $(7,7)$ & $(16,7)$ & $(30,3)$ & $(17,2)$ & $(18,5)$ & $(100)$ \\
\hline \multirow[t]{2}{*}{ Total } & $\mathrm{n}$ & 1428 & 1445 & 2565 & 5072 & 2736 & 3194 & 16440 \\
\hline & $\%$ & $(8,7)$ & $(8,8)$ & $(15,6)$ & $(30,9)$ & $(16,6)$ & $(19,4)$ & $(100)$ \\
\hline
\end{tabular}

Teste Qui-quadrado: $X^{2}=850,499 ; \mathrm{p}<0.001$.

Fonte: Elaboração própria. 
familiar foi de $52,3 \%$ (pouco estresse) e $34,6 \%$ (muito estresse). Já entre os que apontaram o impacto financeiro como o principal durante o isolamento, $55,1 \%$ apresentaram pouco estresse familiar e $23,6 \%$ muito. Percentuais muito próximos aos que citaram o convívio social como principal impacto, $61,7 \%$ relataram pouco estresse familiar e 16,2\% muito. Esse percentual é muito próximo dos dados da relação entre percepção do estresse familiar com a de como o isolamento afeta a renda.

Outros elementos apresentam uma correlação significante com a percepção de estresse familiar em tempos de isolamento social. Em destaque, observou-se: a quantidade de pessoas que estão na mesma habitação, a qualidade da habitação e a expectativa em relação ao tempo de permanência em isolamento. Dentre estes, pode-se destacar que aqueles que afirmaram estar vivenciando situações de estresse familiar são maioria entre os que estão convivendo com uma quantidade maior de pessoas na residência. Embora as diferenças de percentuais a partir de 4 pessoas na residência sejam mínimas (Tabela 3 ).

A mesma situação foi observada na relação entre a qualidade da habitação e o nível de estresse percebido. Para aqueles cuja qualidade da habitação foi percebida como ótima, 13,3\% relataram muito estresse, $52,9 \%$ pouco estresse e $34 \%$ nenhum estresse. Já para aqueles que classificaram a habitação como péssima, 52,4\% relataram muito estresse, $36,2 \%$ pouco estresse e $11,4 \%$ nenhum estresse.

Quando se relacionou a percepção da qualidade da habitação com o tempo que as pessoas estão dispostas a ficar em isolamento, observa- ram-se diferenças entre os estratos. Para aqueles cuja residência foi considerada péssima, $41,9 \%$ ficariam menos de 1 mês e $38 \%$ o tempo necessário. Enquanto, dentre aqueles que consideram a habitação boa ou ótima, $11,53 \%$ ficariam menos de 1 mês e $68,8 \%$ ficariam o tempo necessário (Tabela 4).

$\mathrm{O}$ estresse familiar também parece influenciar na capacidade de manter-se em isolamento social por mais tempo. Aqueles que responderam serem capazes de ficar o tempo que for necessário em isolamento apresentam menores percentuais de muito estresse familiar, apenas $12,25 \%$.

Outro fator que merece destaque é a relação entre a percepção sobre a qualidade do sono e o estresse familiar, pois ambos podem ser um indicativo de impacto na saúde durante o isolamento. Nesse ponto, observou-se que $54,6 \%$ dos que estão dormindo menos estão percebendo muito estresse familiar, enquanto os que estão dormindo a mesma quantidade de horas, $19,1 \%$, percebem muito estresse, e para os que estão dormindo mais horas por dia, $26,2 \%$ percebem que estão tendo muito estresse familiar.

Dentre as pessoas que declararam a qualidade da habitação como ótima, $47 \%$ estão fazendo atividades físicas e $53 \%$ não. Já dentre aqueles que declararam as condições de habitação como ruim ou péssima, $73 \%$ não estão fazendo atividades físicas e $23 \%$ sim. A situação é similar quando se observam as diferentes faixas de renda. Dentre aqueles que se dizem sem renda, apenas $32 \%$ estão fazendo atividades físicas, enquanto $50 \%$ das pessoas que estão na faixa de renda com mais de 8 salários mínimos estão fazendo atividades físicas.

Tabela 3. Pessoas respondentes de 18 anos e mais, pelo número de pessoas na residência, segundo a percepção do grau de estresse familiar.

\begin{tabular}{|c|c|c|c|c|c|c|c|c|c|c|}
\hline \multirow{2}{*}{\multicolumn{2}{|c|}{$\begin{array}{l}\text { Estresse } \\
\text { Familiar }\end{array}$}} & \multicolumn{9}{|c|}{ Quantidade de Pessoas na residência } \\
\hline & & \multirow{2}{*}{$\frac{1}{647}$} & \multirow{2}{*}{$\begin{array}{l}2 \\
1375\end{array}$} & \multirow{2}{*}{$\begin{array}{c}3 \\
1124\end{array}$} & \multirow{2}{*}{$\begin{array}{l} \\
772\end{array}$} & \multirow{2}{*}{$\begin{array}{l}\mathbf{5} \\
303\end{array}$} & \multirow{2}{*}{$\frac{6}{117}$} & \multirow{2}{*}{$\begin{array}{l}\frac{7}{53} \\
53\end{array}$} & \multirow{2}{*}{$\begin{array}{r}\begin{array}{r}8 \text { ou } \\
\text { mais }\end{array} \\
30\end{array}$} & \multirow{2}{*}{$\begin{array}{c}\text { Total } \\
4421\end{array}$} \\
\hline Nenhum & $\mathrm{n}$ & & & & & & & & & \\
\hline & $\%$ & $(14,6)$ & $(31,1)$ & $(25,4)$ & $(17,5)$ & $(6,9)$ & $(2,6)$ & $(1,2)$ & $(0,7)$ & $(100)$ \\
\hline \multirow[t]{2}{*}{ Pouco } & $\mathrm{n}$ & 649 & 2241 & 2574 & 2258 & 898 & 339 & 134 & 87 & 9180 \\
\hline & $\%$ & $(7,1)$ & $(24,4)$ & $(28)$ & $(24,6)$ & $(9,8)$ & $(3,7)$ & $(1,5)$ & $(0,9)$ & $(100)$ \\
\hline \multirow[t]{2}{*}{ Muito } & $\mathrm{n}$ & 204 & 548 & 788 & 698 & 390 & 118 & 52 & 41 & 2839 \\
\hline & $\%$ & $(7,2)$ & $(19,3)$ & $(27,8)$ & $(24,6)$ & $(13,7)$ & $(4,2)$ & $(1,8)$ & $(1,4)$ & (100) \\
\hline \multirow[t]{2}{*}{ Total } & $\mathrm{n}$ & 1500 & 4164 & 4486 & 3728 & 1591 & 574 & 239 & 158 & 16440 \\
\hline & $\%$ & $(9,1)$ & $(25,3)$ & $(27,3)$ & $(22,7)$ & $(9,7)$ & $(3,5)$ & $(1,5)$ & (1) & (100) \\
\hline
\end{tabular}

Teste Qui-quadrado: $X^{2}=497,409 ; \mathrm{p}<0.001$.

Fonte: Elaboração própria. 
Tabela 4. Pessoas respondentes de 18 anos e mais, por tempo a mais que se está disposto a praticar o auto isolamento, segundo a qualidade da residência.

\begin{tabular}{|c|c|c|c|c|c|c|}
\hline \multirow{2}{*}{\multicolumn{2}{|c|}{$\begin{array}{l}\text { Qualidade da } \\
\text { residência }\end{array}$}} & \multicolumn{5}{|c|}{ Tempo a mais que se está disposto a praticar o auto-isolamento } \\
\hline & & \multirow{2}{*}{$\begin{array}{c}\begin{array}{c}\text { Menos de } 1 \\
\text { mês }\end{array} \\
44\end{array}$} & \multirow{2}{*}{$\begin{array}{c}\text { De } 1 \text { a } 2 \text { meses } \\
16\end{array}$} & \multirow{2}{*}{$\begin{array}{c}\begin{array}{c}\text { Mais de } 2 \\
\text { meses }\end{array} \\
5\end{array}$} & \multirow{2}{*}{$\begin{array}{c}\begin{array}{c}\text { Tempo } \\
\text { necessário }\end{array} \\
40\end{array}$} & \multirow{2}{*}{$\begin{array}{r}\text { Total } \\
105\end{array}$} \\
\hline Péssima & $\mathrm{n}$ & & & & & \\
\hline & $\%$ & $(41,9)$ & $(15,2)$ & $(4,8)$ & $(38,1)$ & $(100)$ \\
\hline \multirow[t]{2}{*}{ Ruim } & $\mathrm{n}$ & 110 & 63 & 15 & 156 & 344 \\
\hline & $\%$ & $(32)$ & $(18,3)$ & $(4,4)$ & $(45,3)$ & $(100)$ \\
\hline \multirow[t]{2}{*}{ Regular } & $\mathrm{n}$ & 588 & 559 & 67 & 1251 & 2465 \\
\hline & $\%$ & $(23,9)$ & $(22,7)$ & $(2,7)$ & $(50,8)$ & (100) \\
\hline \multirow[t]{2}{*}{ Boa } & $\mathrm{n}$ & 1158 & 1515 & 236 & 4232 & 7142 \\
\hline & $\%$ & $(16,2)$ & $(21,2)$ & $(3,3)$ & $(59,3)$ & (100) \\
\hline \multirow[t]{2}{*}{ Ótima } & $\mathrm{n}$ & 736 & 1066 & 191 & 4389 & 6382 \\
\hline & $\%$ & $(11,5)$ & $(16,7)$ & (3) & $(68,8)$ & $(100)$ \\
\hline \multirow[t]{2}{*}{ Total } & $\mathrm{n}$ & 2636 & 3219 & 514 & 10068 & 16438 \\
\hline & $\%$ & (16) & $(19,6)$ & $(3,1)$ & $(61,2)$ & $(100)$ \\
\hline
\end{tabular}

Teste Qui-quadrado: $X^{2}=459,444 ; \mathrm{p}<0.001$.

Fonte: Elaboração própria.

\section{Discussão}

Os resultados gerais revelam questões que confirmam o que vem sendo discutido na mídia e nos primeiros estudos e pesquisas publicadas no Brasil referentes ao isolamento social no contexto da pandemia da COVID-199,16. Uma pesquisa realizada entre os dias 3 e 4 de abril mostra resultados parecidos, na qual $94 \%$ dos entrevistados estavam em algum tipo de isolamento, entretanto considerou-se também isoladas pessoas que estavam saindo de casa para visitar amigos e familiares ${ }^{19}$, variável não considerada nesta pesquisa.

$\mathrm{O}$ que se tem observado é que o impacto da pandemia de coronavírus na economia mundial está sendo expressivo, e em especial no Brasil. Dados recentes mostram que já está havendo um aumento significativo no desemprego, 19\% das pessoas entrevistadas afirmaram que já estavam desempregados antes da pandemia. Num primeiro momento já com a pandemia no país, $22 \%$ declararam estar sem trabalhar e, mais recentemente, o número cresceu para $26 \%$, ou seja, um quarto dos respondentes ${ }^{19}$. Um percentual próximo ao encontrado pelos respondentes desta pesquisa informaram ter perdido sua renda.

Outro campo afetado com o isolamento social é o da saúde. O estresse é apontado como uma das principais consequências do isolamento social ${ }^{20,21}$. Pelos dados apresentados, observou-se que $73 \%$ das pessoas que participaram da pesquisa relataram algum grau de estresse em fun- ção da situação do isolamento social, o que sugere a necessidade de se criar ações de comunicação específica para mitigar esse problema.

Uma das influências do estresse na vida das pessoas refere-se às alterações no sono ${ }^{22}$. E os resultados mostraram que para $67 \%$ das pessoas houve uma modificação na rotina do sono, na qual alguns indivíduos estão dormindo mais horas por dia e outros menos.

Outra variável importante relacionada à saúde e ao bem estar das pessoas é a prática de atividades físicas. Com o contexto do isolamento social, realizar exercícios físicos tornou-se um desafio. Os dados da pesquisa mostraram que o percentual de pessoas que estão conseguindo praticar alguma atividade física é de $40 \%$, número compatível com a média nacional de $38 \%$ de pessoas que num contexto de normalidade fazem alguma atividade física ${ }^{23}$.

A média de 3,2 pessoas por domicílio durante o isolamento aproximou-se da nacional de 3,3 habitantes por domicílios ${ }^{24}$. Associada à quantidade de pessoas por domicílio, outra variável muito importante em tempos de isolamento social é a qualidade das habitações, pois o Brasil é um país muito diverso com condições de habitabilidade bastante desiguais, logo, o conforto e a estrutura que esse espaço tem pode fazer muita diferença, entre está ou não em isolamento, mas também nas condições desse isolamento.

Os resultados da pesquisa também revelaram que a maioria das pessoas está muito disposta a 
ficar em casa o tempo que for necessário para enfrentar a pandemia. Dado semelhante foi relatado na Itália, onde $67,5 \%$ afirmaram que continuariam o tempo necessário no autoisolamento, caso o governo prorrogasse a lei que instituiu a prática no país ${ }^{14}$. Dados, como esses, poderiam ser monitorados periodicamente, pois esta pesquisa foi realizada durante o primeiro mês do isolamento, havendo uma tendência à saturação desta condição com o passar do tempo. Ou seja, os dados refletem apenas o retrato do momento, com pessoas que estavam em isolamento por no máximo por vinte dias, diferença da data da coleta de dados (06 a 08 de abril de 2020) com os primeiros decretos de isolamento social da população (16 de março de 2020).

A grande adesão ao isolamento, por parte dos inquiridos, pode ter alguma relação com o medo de se infectar e de sofrer prejuízos a saúde e financeiros ainda maiores. Participantes de estudos anteriores, sobre situações de surtos epidêmicos que exigiram quarentena, relataram temores sobre sua própria saúde e medo de infectar outras pessoas, em especial os membros da família. Esse medo era maior entre os que praticavam a quarentena do que aqueles que não estavam em quarentena ${ }^{21}$.

Mesmo com tanta informação sobre a importância do isolamento no controle da pandemia, um percentual de 7,88\% ainda duvida dessa estratégia. Isso ressalta a importância de que se fortaleçam as campanhas de promoção ao autoisolamento e que sejam combatidas as informações falsas que contradizem e questionam a estratégia de isolamento social.

A análise de diferentes estudos relacionados a surtos e epidemias mostra que os respondentes citaram informações fracas das autoridades de saúde pública como estressores, que trazem pouca segurança sobre as ações a serem tomadas e geram confusão sobre o objetivo da quarentena. Essa confusão decorre das diferenças de estilo, abordagem e conteúdo de várias mensagens das autoridades públicas e à fraca coordenação entre as várias jurisdições e níveis de governo envolvidos, situação semelhante à vivenciada no Brasil durante a pandemia. Destacou-se nesses estudos, certa falta de transparência por parte dos funcionários da saúde e do governo sobre a gravidade da pandemia ${ }^{21}$.

A aparente contradição dos que não estão isolados de acreditarem que a medida de isolamento reduzirá o número de vítimas, pode ser explicada pelo fato de que o isolamento social está causando impactos na renda dessas pessoas, que lhes impedem de praticar o isolamento. Esse fato corrobora com os achados sobre a população inglesa de menor renda, que desejaria estar em isolamento durante a pandemia, mas tem essa possibilidade diminuída em até três vezes em relação aos segmentos de mais alta renda ${ }^{15}$. Isso ressalta a importância das políticas de transferência de renda para a parcela da população que não pode se isolar, como forma de ampliar a estratégia de combate a pandemia, ao mesmo tempo que minimiza o impacto ao bem-estar social.

A perda financeira durante a quarentena é um problema socioeconômico sério e mais um fator de risco para os sintomas de distúrbios psicológicos que podem durar vários meses após a quarentena. E ainda que já estejam em curso medidas de apoio do governo, em alguns casos, a quantia que é recebida torna-se insuficiente ou chega tarde demais, levando as pessoas a ficarem dependentes de suas famílias, o que tende a gerar conflitos entre familiares ${ }^{25,26}$. Estudos anteriores mostram que ter suprimentos básicos inadequados (por exemplo, comida, água, roupas ou acomodação) durante a quarentena era uma fonte de frustração e continuava associado à ansiedade e à raiva 4-6 meses após o fim do isolamento ${ }^{27,28}$.

Apesar de um indicativo, a amostra não captou fielmente a realidade de cerca de 13 milhões de brasileiros que vivem em assentamentos precários ${ }^{6}$. Para esses, as condições de habitação impõem claras limitações ao isolamento social e a adoção das medidas de higiene apontadas pelas organizações sanitárias como essenciais para evitar a contaminação pelo vírus. Portanto, o confinamento domiciliar a partir dessas condições requer medidas complementares por parte dos governos visando garantir padrões mínimos de higiene, salubridade e bem-estar 6 .

Além do impacto na renda, especialmente nos segmentos mais pobres, constatou-se que o impacto no convívio social foi destacado pelo grupo de maior renda. Essa é uma situação observada em outros casos parecidos, onde o confinamento levou à perda da rotina habitual e a um contato social e físico reduzido com outras pessoas, causando tédio, frustração e uma sensação de isolamento em relação ao resto do mundo ${ }^{20,21}$.

Como consequência desse processo o estresse tende a aumentar na população, pois apesar de um tempo relativamente curto já ser capaz de causar impactos à saúde mental, evidências mostram que os impactos psicológicos negativos são maiores se as autoridades determinarem um período previsto mais curto de isolamento e depois aumentarem esse período. Assim, seria menos 
estressante se as autoridades estabelecessem um período maior de isolamento, e caso houvesse melhoras fosse posteriormente reduzido ${ }^{14}$.

Durante o período de quarentena, o efeito psicológico negativo não é surpreendente, mas a evidência de que este ainda possa ser detectado meses ou anos depois é mais preocupante e sugere a necessidade de garantir que medidas eficazes de mitigação sejam implementadas como parte de o processo de planejamento ${ }^{21}$.

Outro dado relevante mostrou que as pessoas com menor renda e em habitações mais precárias estão realizando menos atividades físicas que as do grupo de pessoas de maior renda e melhores condições habitacionais. Isso ressalta o fato de que as pessoas de menor renda, além de mais expostas aos problemas financeiros ocasionados pela pandemia da COVID-19, também estão mais vulneráveis a serem afetadas por problemas de saúde física e psicológicos associados à reclusão necessária no período de isolamento social.

Esses elementos que impactam na percepção e na intenção de adotar o autoisolamento levam a preocupação de por quanto tempo as pessoas continuarão permanecendo isoladas e quais medidas podem ser tomadas para contribuir na redução dos impactos, sejam financeiros, ou na saúde física e mental.

Os dados revelados e discutidos nesta pesquisa, apesar de terem sido coletados em todos os estados brasileiros e em diversificados segmentos da população, apresentam como principal limitação a conveniência da amostra, que ficou sujeita a viés de seleção, na qual se observou uma discrepância entre a representação dos indivíduos de maior renda e escolaridade, maioria na amostra, em relação aos indivíduos de menor renda e escolaridade, minoria na amostra. Desta forma, o estudo não é representativo do comportamento da população brasileira como um todo, mas apenas do universo amostrado.

\section{Considerações finais}

Até o dia 20 de abril, o Brasil já havia registrado mais de 39 mil casos confirmados e 2.507 mortes pela COVID-19. Porém, estimativas mostram que o número real de contaminados pode variar entre nove e quinze vezes mais que os casos notificados ${ }^{29,30}$. Além disso, dados recentes de projeções mostram que surtos recorrentes da $\mathrm{CO}$ -
VID-19 nas estações mais frias provavelmente ocorrerão após a onda pandêmica inicial mais severa. Para evitar que essa situação leve à saturação da capacidade hospitalar, a estratégia de isolamento social prolongado ou intermitente pode ser necessário até $2022^{31}$.

Diante desse quadro é necessário o melhor entendimento possível de como a estratégia de isolamento social é percebida pela sociedade e quais os impactos dessa estratégia na vida das pessoas. Assim, também, investigar diferentes formas de ação para que o isolamento afete menos o bem-estar social e a condição financeira das pessoas, sendo um desafio a ser enfrentado daqui para frente.

Importante também, identificar como os impactos do isolamento se refletem nos diversos segmentos da sociedade, seja em função da renda, sexo, escolaridade, condições de habitação etc. Essa pesquisa buscou fazer isso, ao estabelecer algumas correlações entre variáveis que podem guiar diferentes estratégias para distintos públicos. É notório, e os dados também revelaram, que as populações mais pobres já estão sofrendo um impacto maior do isolamento, especialmente em relação à renda.

Mesmo diante da vulnerabilidade social que a pandemia tem gerado, um ponto chave para seu enfrentamento é a diminuição da circulação de pessoas nas ruas e nos espaços públicos coletivos. Os dados da pesquisa mostraram que a maioria dos respondentes está contribuindo com esse propósito, pois acreditam que a estratégia do isolamento será eficaz para evitar o colapso na assistência hospitalar e a redução no número de vítimas da COVID-19. Isso remete à urgência de medidas de proteção social e suporte financeiro, prioritariamente para os segmentos sociais ainda mais vulnerável nesse momento de crise.

\section{Colaboradores}

ACV Bezerra trabalhou no desenho da pesquisa, na concepção do texto, nas análises dos dados e na redação final. CEM Silva trabalhou no desenho da pesquisa, na concepção do texto, na análises dos dados e na redação final. FRG Soares trabalhou na análise dos dados. JAM Silva fez a revisão crítica do trabalho. 


\section{Agradecimentos}

Agradecemos a todos e todas que gentilmente responderam e compartilharam o questionário em suas redes sociais, especialmente aos grupos de pesquisas parceiros que difundiram o questionários em seus respectivos estados.

\section{Referências}

1. Wu F, Zhao S, Yu B, Chen YM, Wang W, Song ZG, Hu Y, Tao ZW, Tian JH, Pei YY, Yuan ML, Zhang YL, Dai FH, Liu Y, Wang QM, Zheng JJ, Xu L, Holmes EC, Zhang YZ. A new coronavirus associated with human respiratory disease in China. Nature 2020; 579(7798):265-269.

2. European Center for Disease Prevention and Control (ECDC). Coronavirus disease 2019 (COVID-19) pandemic: increased transmission in the EU/EEA and the UK - seventh update. Stockholm: ECDC; 2020. [acessado 2020 Abr 14]. Disponível em: https://www. ecdc.europa.eu/sites/default/files/documents/RRAseventh-update-Outbreak-of-coronavirus-diseaseCOVID-19.pdf

3. World Health Organization (WHO). Rolling updates on coronavirus disease (COVID-19) 2020. Geneva: WHO; 2020. [acessado 2020 Abr 14]. Disponível em: https://www.who.int/emergencies/diseases/novel-coronavirus 2019/events-as-they-happen.

4. Croda JHR, Garcia LP. Immediate Health Surveillance Response to COVID-19 Epidemic. Epidemiol. Serv. Saúde 2020; 29(1):e2020002.

5. Rodriguez-Morales AJ, Gallego V, Eescalera-Antezana JP, Méndez CA, Zambrano LI, Franco-Paredes C, Suárez JA, Rodriguez-Enciso HD, Balbin-Ramon GJ, Savio-Larriera E, Risquez A, Cimerman S. COVID-19 in Latin America: The implications of the first confirmed case in Brazil. Travel Medicine and Infectious Disease 2020; 10.1016 .

6. Pires RRC. Os efeitos sobre grupos sociais e territórios vulnerabilizados das medidas de enfrentamento à crise sanitária da covid-19: propostas para o aperfeiçoamento da ação pública: Nota Técnica. Brasília: IPEA; 2020. [acessado 2020 Abr 14]. Disponível em: http://www. ipea.gov.br/portal/index.php?option=com_alphacontent\&view $=$ alphacontent $\&$ Itemid $=357$

7. Farias HSF. O avanço da Covid-19 e o isolamento social como estratégia para redução da vulnerabilidade, Espaço e Economia. [Online], 2020, posto online no dia 08 abril 2020. [acessado 2020 Abr 17]. Disponível em: http://journals.openedition.org/espacoeconomia $/ 11357$

8. Hale T, Webster S. Oxford COVID-19 Government Response Tracker. Data use policy: Creative Commons Attribution CC BY standard. 2020. [acessado 2020 Abr 14]. Disponível em: https://www.bsg.ox.ac.uk/ research/publications/variation-government-responses-covid-19

9. Datafolha. Opinião sobre a pandemia do Coronavírus: comportamento da população e medidas do governo. São Paulo: Datafolha; 2020. [acessado 2020 Abr 14]. Disponível em: http://media.folha.uol.com. br/datafolha/2020/04/06/6c9855d692b869f13c5d83c421568342hb.pdf

10. Garcia LP, Duarte E. Intervenções não farmacológicas para o enfrentamento à epidemia da COVID-19 no Brasil. Epidemiologia e Serviços de Saúde 2020; 29(2):e2020222. 
11. Ornell F, Schuch JB, Sordi AO, Kessler FHP. Pandemia de medo e COVID-19: impacto na saúde mental e possíveis estratégicas. Revista Debates in Psychiatry. No prelo 2020. [acessado 2020 Abr 14]. Disponível em: http://www.ufrgs.br/ufrgs/noticias/arquivos/pandemia-de-medo-e-covid-19-impacto-na-saude-mental-e-possiveis-estrategias

12. Porsse AA, Souza KB, Carvalho TS, Vale VA. Impactos Econômicos do COVID-19 no Brasil. Nota Técnica NEDUR-UFPR No 01-2020. Curitiba: Núcleo de Estudos em Desenvolvimento Urbano e Regional (NEDUR) da Universidade Federal do Paraná; Abril/2020. [acessado 2020 Abr 14]. Disponível em: http://www.nedur. ufpr.br/portal/wp-content/uploads/2020/04/notatecnica-nedur-ufpr-01-2020-impactos-economicosda-covid-19-no-brasil.pdf

13. World Healh Organization (WHO). Coronavirus disease 2019 (COVID-19): situation report, 72. Geneva: WHO; 2020. [acessado 2020 Abr 14]. Disponível em: https://www.who.int/emergencies/diseases/novel-coronavirus-2019/situation-reports

14. Briscese G, Lacetera N, Macis SM, Tonin M. Compliance with covid-19 social-distancing measures in italy: the role of expectations and duration. Cambridge: NBER Working Paper Series; 2020. [acessado 2020 Abr 14]. Disponível em: http://www.nber.org/papers/ w26917

15. Atchimson C, Bowman L, Vrinten C, Redd R, Pristera P, Eaton JW, Ward H. Perceptions and behavioural responses of the general public during the COVID-19 pandemic: A cross-sectional survey of UK Adults. medRxiv 2020; 04.01.20050039.

16. Lima DLF, Dias AA, Rabelo RS, Cruz ID, Costa SC, Nigri FMN, Neri JR. COVID-19 no Estado do Ceará: Comportamentos e crenças na chegada da pandemia. Cien Saude Colet 2020; 25(5):1575-1586.

17. Brasil. Ministério da Saúde (MS). Conselho Nacional de Saúde. Resolução no 510, de 7 de abril de 2016. Dispõe sobre as normas aplicáveis a pesquisas em Ciências Humanas e Sociais. Diário Oficial da União 2016; 24 maio.

18. Gil AC. Como elaborar projetos de pesquisa. $6^{\circ}$ ed. São Paulo: Editoras Atlas; 2019.

19. Opinion Box. Impacto nos hábitos de compra e consumo: como a pandemia está transformando o comportamento do consumidor brasileiro. Nota Técnica 2. Belo Horizonte: Opinion Box Pesquisas; 2020. [acessado 2020 Abr 14]. Disponível em: https://blog. opinionbox.com/dados-atualizados-sobre-o-coronavirus/

20. Van Bavel Jj, Boggio P, Capraro V, Cichocka A, Cikara M, Crockett M, Willer R. Using social and behavioural science to support COVID-19 pandemic response. [acessado 2020 Mar 24]. Disponível em: https://doi. org/10.31234/osf.io/y38m9

21. Brooks SK, Webster Rk, Smith Le, Woodland L,Wessely S, Greenberg N, Rubin Gj. The psychological impact of quarantine and how to reduce it: rapid review of the evidence. Lancet 2020; 395(10227):912-920.
22. Van Reeth, O. Weibel L, Spiegel K, Leproult R, Dugovic $\mathrm{C}$, Maccaric $\mathrm{S}$. Interactions between stress and sleep: from basic research to clinical situations. Sleep Medicine Reviews 2000; 4(2):201-220.

23. Instituto Brasileiro de Geografia e Estatística (IBGE). Pesquisa nacional por amostra de domicílios: síntese de indicadores 2015. Rio de Janeiro: IBGE; 2016. [acessado 2020 Abr 14]. Disponível em: https://biblioteca. ibge.gov.br/visualizacao/livros/liv98887.pdf

24. Instituto Brasileiro de Geografia e Estatística (IBGE). Censo Demográfico 2010 - Características da população e dos domicílios: resultados do universo. Rio de Janeiro: IBGE; 2011. [acessado 2020 Abr 14]. Disponível em: https://biblioteca.ibge.gov.br/visualizacao/periodicos/93/cd_2010_caracteristicas_populacao_domicilios.pdf

25. Bai YM, Lin CC, Lin CY, Chen JY, Chue CM, Chou P Survey of stress reactions among health care workers involved with the SARS outbreak. Psychiatric Services 2004; 55(9):1055-1057.

26. Liu X, Kakade M, Fuller Cj, Fan B, Fang Y, Kong J. Guan Z, Wu P. Depression after exposure to stressful events: Lessons learned from the severe acute respiratory syndrome epidemic. Comprehensive Psychiatry 2012; 53(1):15-23.

27. Blendon, RJ Benson JM, DesRoches CM, Raleigh E, Taylor-Clark K. The Public's Response to Severe Acute Respiratory Syndrome in Toronto and the United States. Clinical Infectious Diseases 2004; 38(7):925-931.

28. Wilken J, Pordell P, Goode B, Jarteh R, Miller Z, Saygar, BG, Maximore L, Borbor Wm, Carmue M, Walker Gw, Yeiah A. Knowledge, attitudes, and practices among members of households actively monitored or quarantined to prevent transmission of ebola virus disease-margibi county, liberia: February-march 2015. Prehospital and Disaster Medicine 2017; 32(6):673-678.

29. Batista A, Antunes B, Faveret G, Peres I, Marchesi J, Dantas L, Bastos L, Aguilar S, Ranzani O, Baião F, Maçaira P, Hamacher S, Carnevale R, Bozza, F. Projeção de casos de infecção por COVID-19 no Brasil até 30 de março de 2020: Nota Técnica. Rio de Janeiro: Núcleo de Operações e Inteligência em Saúde (NOIS); 2020. [acessado 2020 Abr 14]. Disponível em: http:// www.saude.mppr.mp.br/arquivos/File/Corona/NT4 NOIS_PUCRIO.pdf

30. Portal COVID-19 Brasil. Análise Subnotificação. [acessado 2020 Abr 13]. Disponível em: https://ciis.fmrp. usp.br/COVID19/analise-subnotificacao

31. Kissler SM, Tedijanto C, Goldestein E, Grad YH, Lipsitch M. Projecting the transmission dynamics of SAR$\mathrm{S}-\mathrm{CoV}-2$ through the postpandemic period. Science 2020; pii:eabb5793.

Artigo apresentado em 18/04/2020

Aprovado em 21/04/2020

Versão final apresentada em 23/04/2020 
